\title{
Asteroseismology and evolution of GW Vir stars
}

\author{
P.-O. Quirion \\ Aarhus Universitet, Institut for Fysik og Astronomi, Ny Munkegade Bld. 1520, DK-8000 Aarhus, Danmark
}

\begin{abstract}
We present a summary of current knowledge of the hot, pulsating, hydrogen deficient GW Vir stars. At $T_{\text {eff }} \simeq 120,000 \mathrm{~K}$ and with an atmosphere composed mainly of helium, carbon, and oxygen, these pulsating stars are showing multiperiodic, intermediate- to high-order g modes. They include in their class some of the hottest white dwarfs and planetary nebulae. We present non-adiabatic asteroseismology results used to determine the atmospheric parameters of GW Vir stars. We also discuss some of the latest asteroseismology results using state of the art post-Asymptotic Giant Branch evolution models.
\end{abstract}

Individual Objects: Longmore 4, Abell 43, NGC 7094, NGC 246, NGC 5189, Sk 3, NGC 2867, NGC 6905, NGC 1501, HE 1429-1209, RX J2117.1+3412, HS 2324+3944, NGC 2371, K1-16, PG 1159-035, VV 47, PG 2131+066, PG 1707+427, PG 0122+200, H1504+65

\section{Introduction}

From $T_{\text {eff }} \sim 85000 \mathrm{~K}$ to $200000 \mathrm{~K}$ and from $\log g=5.5$ to 8.0 extends a region of the $\log g-T_{\text {eff }}$ diagram seated exactly on the "knee" leading from the post-Asymptotic Giant Branch (post-AGB) to the white dwarf cooling track. This region is filled with a variety of hydrogen deficient stars being referred to, more and more in recent articles and conferences, as pre-white dwarfs. A multitude of spectral and sub-spectral types are included under that generic name. We can regroup them under three main types. The PG 1159 stars, the Early type Wolf Rayet Central Stars of Planetary Nebulae, or more conveniently the [WCE] stars, and the $\mathrm{O}(\mathrm{He})$ stars.

The [WCE] and PG 1159 stars are both believed to be the products of the born-again scenario in which hydrogen rich stars entering the white dwarf cooling track are pushed back to the AGB by a (very)-late thermal pulse (Iben et al. 1983, Herwig et al. 1999). At the end of the born-again process, the star moves back toward the white dwarf branch and is highly or completely depleted of hydrogen. At this point, it shows a significant amount of helium, carbon and oxygen in its spectra. The range of abundance is $X(\mathrm{He}) \simeq 0.30-0.85$, $X(C) \simeq 0.15-0.60$, and $X(O) \simeq 0.02-0.20$, by mass. There is also the peculiar object $\mathrm{H} 1504+65$ with its nearly pure carbon and oxygen spectra $\mathrm{X}(\mathrm{C}) \simeq \mathrm{X}(\mathrm{O}) \simeq 0.50$, and the so called hybrid stars which show a relatively large amount of hydrogen, $X(H) \simeq 0.15-0.35$. More details about abundance patterns and the evolutionary history of these stars are found in Werner \& Herwig (2006). The origin of $\mathrm{O}(\mathrm{He})$ stars is somewhat more nebulous. They show high helium enrichment with only traces of the CNO elements in their spectra with composition of $X(H) \simeq 0.10-0.50 \times(\mathrm{He}) \simeq 0.50-0.90$. We refer the reader to Rauch et al. (2008) and references therein for more details. In any case, the fate of all pre-white dwarfs is to become white dwarfs. Those that are completely hydrogen depleted should turn into 
Table 1: Confirmed GW Vir stars.

\begin{tabular}{lcccl}
\hline Star & $\log g$ & $T_{\text {eff }}[k K]$ & Periods [s] & Remark \\
\hline Longmore 4 & 5.5 & 120 & $831-2325$ & transient \\
Abell 43 & 5.7 & 110 & $2604-5529$ & hybrid \\
NGC 7094 & 5.7 & 110 & $2000-5000$ & hybrid \\
NGC 246 & 5.7 & 150 & $480-4560$ & \\
NGC 5189 & 6.0 & 135 & 690 & [WCE] \\
Sk 3 & 6.0 & 140 & $929-2183$ & [WCE] \\
NGC 2867 & 6.0 & 141 & 769 & [WCE] \\
NGC 6905 & 6.0 & 141 & $710-912$ & [WCE] \\
NGC 1501 & 6.0 & 134 & $1154-5235$ & \\
HE 1429-1209 & 6.0 & 160 & 919 & \\
RX J2117.1+3412 & 6.0 & 170 & $694-1530$ & hybrid \\
HS 2324+3944 & 6.2 & 130 & $2005-2569$ & \\
NGC 2371 & 6.3 & 135 & $923-1825$ & \\
K1-16 & 6.4 & 140 & $1500-1700$ & \\
PG 1159-035 & 7.0 & 140 & $339-982$ & \\
V 47 47 & 7.0 & 130 & $261-4310$ & \\
PG 2131+066 & 7.5 & 95 & $339-508$ & \\
PG 1707+427 & 7.5 & 85 & $336-942$ & \\
PG 0122+200 & 7.5 & 80 & $336-612$ & \\
\hline
\end{tabular}

helium rich DO white dwarfs, while the others, with various amounts of hydrogen, should transform into DA white dwarfs.

To this description of the pre-white dwarf region, we add the main topic of this review, which is the GW Vir instability strip. The strip covers a large part of the pre-white dwarf region and is caused by a classical $\kappa$ mechanism triggered by partial ionization of the $\mathrm{K}$-shell of both carbon and oxygen (Starrfield et al. 1983, Starrfield et al. 1984, Cox 2003, Quirion et al. 2007a). We have observed 19 [WCE] and PG 1159 stars in the GW Vir instability strip. These pulsating stars are showing medium to high radial order g modes with $k \gtrsim 15$ and $I=1,2$. None of the $\mathrm{O}(\mathrm{He})$ stars is known to be variable. Only small traces of carbon are detected in the spectra of one of the four $\mathrm{O}(\mathrm{He})$ stars, not enough to trigger oscillations and make it part of the GW Vir instability strip. Table 1 presents an updated list of the GW Vir stars with their stellar parameters and detected instability range. References for this table can be found in Quirion et al (2007a), except for VV 47 (González Pérez et al. 2006) and NGC 7094 (Solheim et al. 2007) which have been discovered to pulsate only recently.

\section{Non-adiabatic studies}

In Quirion et al. (2007a), the position of the blue edge of the GW Vir instability strip is shown to be a function of the exact carbon and oxygen content in the envelope of the pre-white dwarf. A rule of thumb is to state that the higher the content of carbon and oxygen in the pre-white dwarf is, the higher the temperature of its blue edge will be. Since pre-white dwarfs are showing a large range of chemical composition, there is accordingly a large set of possible blue edges. This phenomenon is depicted in Fig. 1. Following that simple idea, two reasons are invoked to explain the stability of a star located in the pre-white dwarf region. First, the depletion of $\mathrm{C}$ and $\mathrm{O}$ in the envelope of the star, which makes the driving of pulsation impossible, and the high temperature of the star that puts it outside the instability strip. The later reason is invoked to explain why $\mathrm{H} 1504+65$, a pure $\mathrm{C}-\mathrm{O}$ but extremely hot $(200000 \mathrm{~K})$ star, is stable.

The stability of pre-white dwarfs is thus determined by the chemical composition of the envelope and the effective temperature. Since these two parameters are fixed by spectroscopy, 


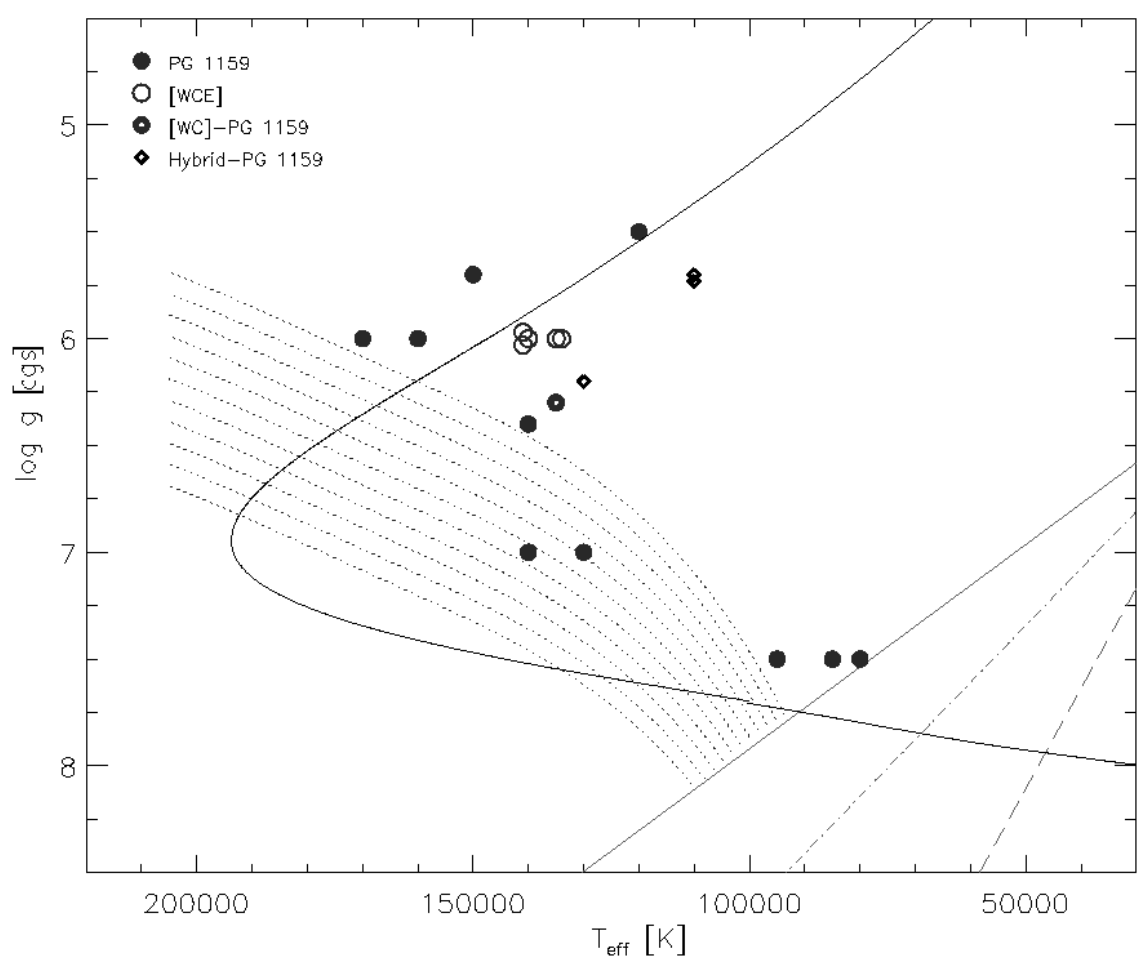

Figure 1: Positions of the GW Vir stars in the $\log g-T_{\text {eff }}$ diagram. The different spectral types are specified by different symbols. The blue dotted curves symbolize the intrinsic fuzziness of the blue edge caused by its dependence on the atmospheric chemical composition. The red lines are the theoretical red edges calculated with full evolutionary models including diffusion and mass loss. Each red edge is calculated with a different mass loss law, $\dot{M}=1.00 \times 10^{-17} L^{2.36}$ refers to the solid red line, $\dot{M}=1.29 \times 10^{-15} L^{1.86}$ refers to the dot-dashed red line and $\dot{M}=1.82 \times 10^{-13} L^{1.36}$ refers to the dashed red line.

one can check the consistency of the spectroscopic measurement by comparing the observed instability range of GW Vir stars with the period range obtained with non-adiabatic computations. Table 2 shows the results of that exercise for a sample of GW Vir stars. One can compare the unstable period ranges obtained by models using the spectroscopic values for the composition and the atmospheric parameters of Table 2 to the observed ranges of Table 1 and state that the matches are qualitatively good. However, we can find better fits to the observed period bands by varying the gravity and effective temperature of the models within observational uncertainties. The results of this exercise, explained in more details in Quirion et al. $(2008,2009)$ is shown in Table 2 under "Non-adiabatic asteroseismology". An explicit computation is shown in Fig. 2 for PG 1159-035, the prototype of the class and the best studied GW Vir star. Non-adiabatic asteroseismology can be considered as a consistency check of spectroscopic measurements. Nonetheless, as Table 2 shows, non-adiabatic asteroseismology also gives a significant improvement to the GW Vir stars' measured surface gravity, and to some extent to their measured effective temperature. 
Table 2: Non-adiabatic asteroseismology.

\begin{tabular}{lcccccc}
\hline & \multicolumn{3}{c}{ Quantitative spectroscopy } & \multicolumn{3}{c}{ Non-adiabatic asteroseismology } \\
Star & $\begin{array}{l}\log g \\
\pm .5 \mathrm{dex}\end{array}$ & $\begin{array}{c}T_{\text {eff }}[\mathrm{kK}] \\
\pm \sim 10 \%\end{array}$ & Periods [s] & $\log g$ & $T_{\text {eff }}[\mathrm{kK}]$ & Periods [s] \\
\hline NGC 246 & 5.70 & 150 & $514-11453$ & $\sim 5.75$ & - & $604-4477$ \\
RX J2117.1+3412 & 6.00 & 170 & $635-2202$ & $\sim 6.10$ & $\sim 180 \pm 10$ & $681-1530$ \\
PG 1159-035 & 7.00 & 140 & $247-623$ & $6.80 \pm .05$ & $144 \pm 3$ & $336-987$ \\
V 47 & 7.00 & 130 & $216-891$ & $6.10 \pm .10$ & $130 \pm 20$ & $235-3531$ \\
PG 2131+066 & 7.50 & 95 & $197-650$ & $7.25 \pm .25$ & - & $118-508$ \\
PG 1707+427 & 7.50 & 85 & $188-765$ & $7.35 \pm .05$ & $81 \pm 5$ & $224-960$ \\
PG 0122+200 & 7.50 & 80 & $198-847$ & $7.50 \pm .30$ & - & $330-602$ \\
\hline
\end{tabular}

\section{Red edge}

The main factor that differentiates PG 1159 from the [WCE] stars is the presence of a stronger radiatively driven wind in the latter. The wind produces wide strong CIV, Hell and OVI emission lines, giving the [WCE] type its characteristic spectral signature. With time and decreasing luminosity, the magnitude of the outgoing wind diminishes, thus weakening the carbon emission lines in the [WCE] stars and letting the PG 1159 features appear in the spectra of the [WC]-PG 1159 transition objects. A further decrease of the mass loss and wind completes the scenario whereby the wide carbon emission lines disappear, leading to a full characteristic PG 1159 spectrum. Hence the sequence [WCE] $\rightarrow$ PG 1159.

The following evolutionary step connects PG 1159 to DO stars, where the spectral type DO belongs to the He-dominated atmosphere white dwarfs. This scenario is also strongly supported by quantitative spectroscopy (Werner \& Herwig 2006). The strong wind present in [WC] and PG 1159 stars tends to homogenize their envelope (see, e.g. Chayer et al. 1997, Unglaub \& Bues 2001). As this wind weakens with lowering luminosity, it progressively looses its homogenizing capacity and gravitational settling takes over. This will cause carbon and oxygen to precipitate while helium will float at the surface of the star. As carbon and oxygen are responsible for the $\kappa$ mechanism in these stars, it is expected that gravitational settling will ultimately bring the driving to a stop, drawing the red edge of the class (Quirion et al. 2007b). The temperature of the red edge is linked to the strength of the mass loss. A weaker mass loss will make the carbon and oxygen sink more rapidly while the stronger wind will keep these elements afloat a longer time and push the red edge to lower temperature. Results from Quirion et al. (2007b), showing the effect of the mass loss strength on the position of the red edge, using full evolutionary calculation including diffusion and mass loss, are pictured in Fig. 1.

\section{Adiabatic studies}

Non-adiabatic processes can be studied with only an approximate knowledge of GW Vir cores. These processes happen above $\log q=\log \left(1-m(r) / M_{\star}\right) \lesssim-6$ and only this region will affect the instability range of these stars. However, GW Vir asteroseismology, which does a one by one comparison between observed modes and those of putative models, is more influenced by the composition and structure below $\log q \gtrsim-6$. As shown in Fig. 8 of the pulsating white dwarf review by Fontaine \& Brassard (2008), the importance of the deeper layers in the construction of a star's eigenmodes increases with temperature on the white dwarf track. The chemical stratification of the core will, unlike the case of DBV and DAV white dwarfs, play an important role in the construction of the GW Vir eigenmodes.

Another factor influencing the structure of GW Vir stars, especially those at low gravity, 


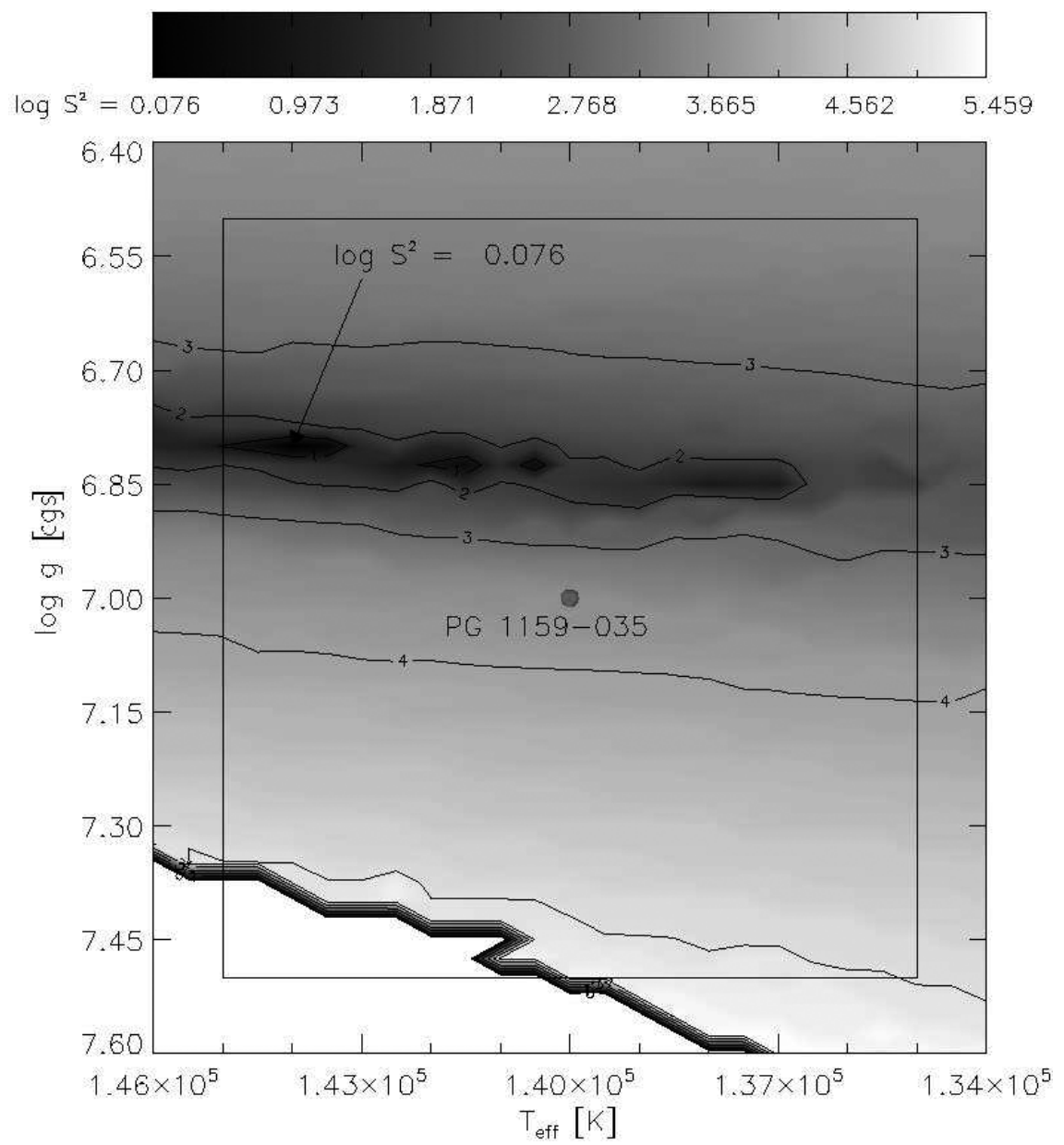

Figure 2: Calculated $S^{2}$ function grid (in logarithmic units) for PG 1159-035 in the $\log g-T_{\text {eff }}$ diagram. The spectroscopy position of the star is shown with a red dot. The box represents its associated uncertainties. The arrow points to the best-fit non-adiabatic model $\left(\log g=6.8\right.$ and $\left.T_{\text {eff }}=144000 \mathrm{~K}\right)$. The $S^{2}$ function used for the fit is described in detail in Quirion et al. $(2008,2009)$.

is their memory of the thermal pulse undergone during the born-again phase. This means that the region where the thermal pulse occurred, around $\log q \sim-2$, can be significantly far from thermal equilibrium. For that reason, the exact treatment of mixing and burning should influence the path of a model in the pre-white dwarf region, and will certainly influence the model eigenmodes (Herwig \& Austin 2004, Miller Bertolami \& Althaus 2007).

Presently, the most sophisticated and extended set of models evolving through the prewhite dwarf region is the one of the La Plata group (Althaus et al. 2005, Miller Bertolami \& Althaus 2006). Their models are starting on the main sequence and ultimately pass through 


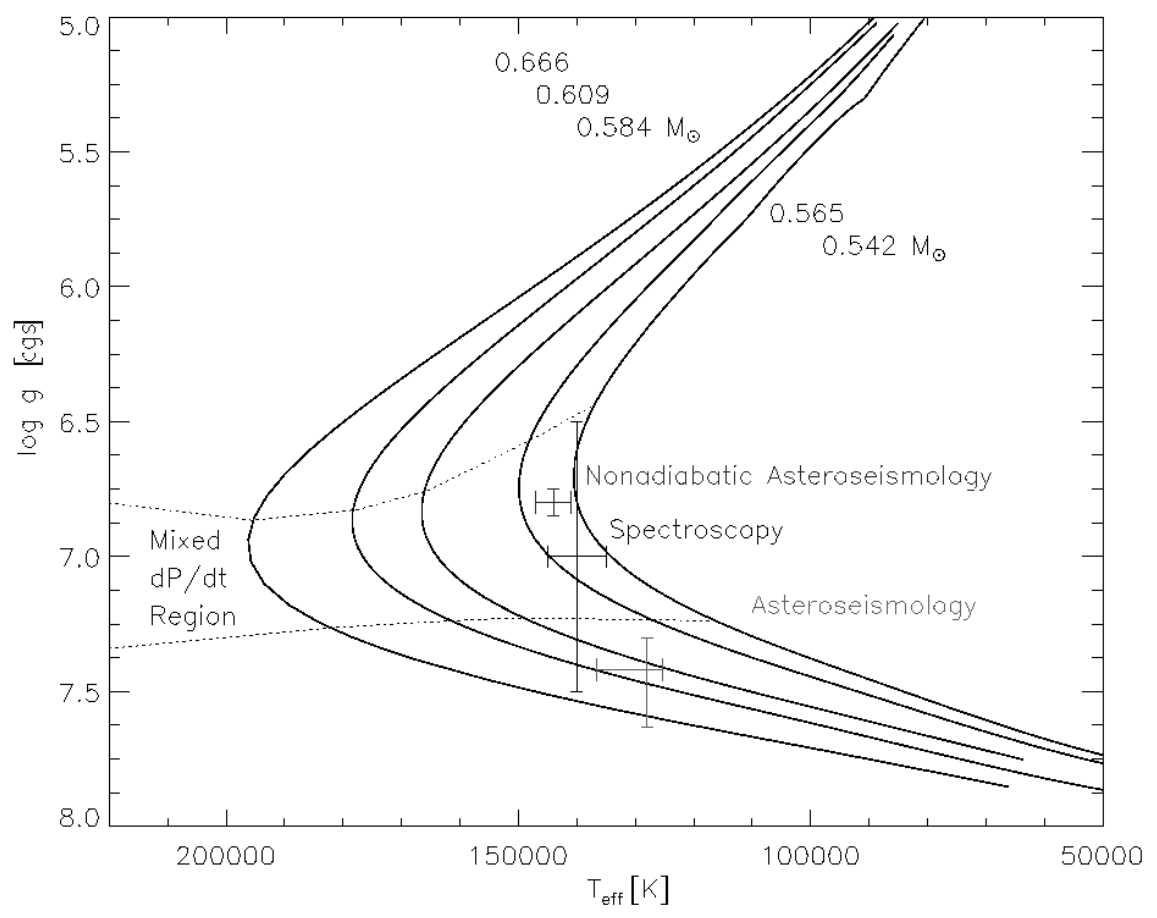

Figure 3: Five pre-white dwarf tracks after undergoing a born-again phase. Enclosed in between the dotted lines are the models showing simultaneously positive and negative (mixed) values of $\dot{P}_{k}$ (Miller Bertolami \& Althaus 2006). Along the tracks are three fits for the star PG 1159-035. The asteroseismology fit (Córsico et al. 2008) is at higher gravity and derived directly from the presented tracks, the spectroscopic fit (Jahn et al. 2007) from non-LTE atmosphere modelling, and the non-adiabatic fit from static envelope models using the chemical composition derived from the spectroscopic fitting (Quirion et al. 2008).

the born-again phase before they reach the pre-white dwarf region. Some of their tracks are reproduced in Fig. 3. An interesting characteristic of the seismology of these tracks is the region where the temporal variation of the period of individual modes $\dot{P}_{k}$ is positive for some of the modes $k$ and negatives for others. This mixed $\dot{P}$ region marks the transition between the post-AGB, where gravitational contraction dominates and $\dot{P}_{k} \sim-d(\log g) / d t \lesssim 0, \forall k$, and the white-dwarf track, where cooling dominates and $\dot{P}_{k} \sim-d T_{\text {eff }} / d t \gtrsim 0, \forall k$.

It its quite remarkable that over 30 years of measurements, we have been able to determine with increasing precision the value of $\dot{P}$ for several modes of PG 1159-035. Even more remarkable is the mixed $\dot{P}$ behavior of its modes. Table 3 shows all measurements with $I=0$ and $m=0$ of $\dot{P}_{k}$ where $k$ is the radial order taken from (Costa \& Kepler 2008).

As mentioned in the caption of Fig. 3, fits for PG 1159-035 from spectroscopy, nonadiabatic asteroseismology, and asteroseismology are superimposed to the tracks. The asteroseismology fit derives directly from the presented tracks (Córsico et al. 2008). Unfortunately, the adiabatic fit is not able to reproduce the mixed $\dot{P}$ behavior of the star. Also, even if they are reasonably close, it seems that spectroscopy and non-adiabatic asteroseismology, which both depend on the structure of the upper part of the star, are at odds with the core-influenced fit of asteroseismology. 
Table 3: $\dot{P}$ rate measurements for the modes of PG $1159-035$ identified as $I=1$ and $m=0$.

\begin{tabular}{ccr}
\hline$P[\mathrm{~s}]$ & $k$ & \multicolumn{1}{r}{$\times 10^{-1}$} \\
\hline 390.3 & 14 & $10.4 \pm 2.3$ \\
452.4 & 17 & $2.6 \pm 0.5$ \\
494.8 & 19 & $-30.1 \pm 2.8$ \\
517.1 & 20 & $18.2 \pm 0.8$ \\
538.1 & 21 & $1.0 \pm 0.7$ \\
558.4 & 22 & $-4.3 \pm 1.9$ \\
\hline
\end{tabular}

The reason of the disparity between the asteroseismological fit and the other aspects of the problem probably rests on the following factors. PG 1159-035 shows medium- to high-order modes with $k \gtrsim 15$. This means that the modes are close to the asymptotic regime where the periods and their spacing depend more on the star's global parameters (Unno et al. 1989) and less on the exact structure of the star. It is therefore reasonable to suggest that a family of solutions and not a single model should be able to yield good asteroseismological fits to PG 1159-035. One could picture this assessment of degenerate solutions by comparing the fits to PG 1159-035 coming from two very different generations of models; the older study of Kawaler \& Bradley (1994) and the modern study of Córsico at al. (2008). Both studies give comparably good asteroseismological fits to the star's eigenfrequencies. We can consider each of the model sets to be a slice of the pre-white dwarf parameter space and state that each of the fits is a solution in the valley of good solutions for PG 1159-035. These are only two studies and the parameter space for asteroseismology (core composition, effect of the last thermal pulse, etc.) is still to be explored in detail. Unfortunately, the construction of modern pre-white dwarf models involves heavy computations.

There is a brighter side to this puzzle. We have in sight the deeper structure of GW Vir stars. One might achieve the goal of understanding these deeper layers by looking at every one of the multiple angles revealed by GW Vir stars, spectroscopy, non-adiabatic processes, asteroseismology, and $\dot{P}$, and try to build a set of models that would fit all these aspects at the same time. From there, we would answer the central problem of stellar astrophysics, which is to know a star from its surface to its core.

Acknowledgments. I would like to thank G. Fontaine for his interest in this work.

\section{References}

Althaus, L. G., Serenelli, A. M., Panei, J. A., et al. 2005, A\&A, 435, 631

Bond, H. E. 2008, ASP Conf. Ser., 391, 129

Chayer, P., Fontaine, G., \& Pelletier, C. 1997, ASSL, 214, 253

Córsico, A. H., Althaus, L. G., Kepler, S. O., et al. 2008 A\&A, 478, 869

Costa, J. E. S., \& Kepler, S. O. 2008, A\&A 489, 1225

Cox, A. N. 2003, ApJ, 585, 975

Fontaine, G., \& Brassard, P. 2008, PASP, 120, 1043

González Pérez, J. M., Solheim, J.-E., \& Kamben, R. 2006, A\&A, 454, 527

Herwig, F., Blöcker, T., Langer, N., \& Driebe, T. 1999, A\&A, 349, L5

Herwig, F., \& Austin, S. M. 2004, ApJ, 613, L73

Iben, I., Jr., Kaler, J. B., Truran, J. W., \& Renzini, A. 1983, ApJ, 264, 605

Jahn, D., Rauch, T., Reiff, E., et al. 2007, A\&A, 426, 281

Kawaler, S. D. \& Bradley, P. A. 1994, ApJ, 427, 415 
Miller Bertolami, M. M., \& Althaus, L. G. 2006, A\&A, 454, 845

Miller Bertolami, M. M., \& Althaus, L. G. 2007, A\&A, 470, 675

Quirion, P.-O., Fontaine, G., \& Brassard, P. 2007a, ApJS, 171, 219

Quirion, P.-O., Fontaine, G., \& Brassard, P. 2007b, ASP Conf. Ser., 372, 649

Quirion, P.-O., Fontaine, G., \& Brassard, P. 2008, J. Phys.: Conf. Ser., 118, 012069

Quirion, P.-O., Fontaine, G., \& Brassard, P. 2009, J. Phys.: Conf. Ser., in press

Rauch, T., Reiff, E., Werner, K., \& Kruk, J. W. 2008, ASP Conf. Ser., 391, 135

Solheim, J.-E., Vauclair, G., Mukadam, A. S., et al. 2007, A\&A, 468, 1057

Starrfield, S. G., Cox, A. N., Hodson, S. W., \& Pesnell, W. D. 1983, ApJ, 268, L27

Starrfield, S., Cox, A. N., Kidman, R. B., \& Pesnell, W. D. 1984, ApJ, 281, 800

Unglaub, K., \& Bues, I. 2001, A\&A, 374, 570

Unno, W., Osaki, Y., \& Ando, H. 1989, in "Nonradial oscillations of stars", University of Tokyo Press

Werner, K., Hamann, W.-R., Heber, U., et al. 1992, A\&A, 259, L69

Werner, K., \& Herwig, F. 2006, PASP, 118, 183

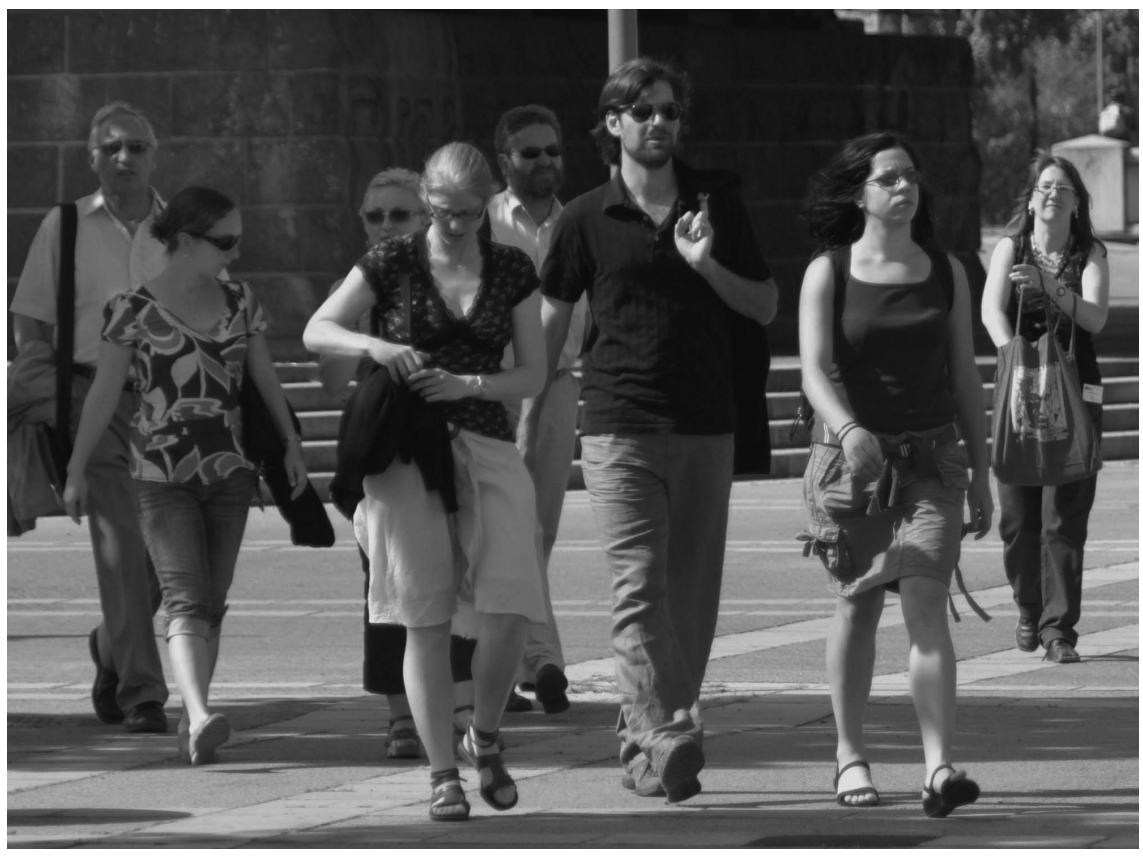

